Supporting Information

\title{
Mechanical-Induced Polarization Switching in Relaxor Ferroelectric Single Crystals
}

Jihong Bian ${ }^{l}$, Yuting Wang ${ }^{1}$, Ren Zhu' ${ }^{2}$, Lei Wang ${ }^{l}$, Bian Yang ${ }^{l}$, Jianwei Wang ${ }^{1}$, Dawei Zhang 3 , Congcong $\mathrm{Xu}^{1}$, Tao $\mathrm{Li}^{4}$, Dwight Viehland ${ }^{5}$, Yaodong Yang ${ }^{1 *}$

1 Frontier Institute of Science and Technology, State Key Laboratory for Mechanical

Behavior of Materials, Xi'an Jiaotong University, Xi'an 710049, China

2 Oxford Instruments, No. 461 Hongcao Road, Shanghai 200233, China

3 School of Materials Science and Engineering, University of New South Wales, Sydney,

New South Wales 2052, Australia

4 Center for Spintronics and Quantum Systems, State Key Laboratory for Mechanical

Behavior of Materials, Department of Materials Science and Engineering, Xi'an Jiaotong

University, Xi'an, Shaanxi 710049, China

5 Department of Materials Science and Engineering, Virginia Tech, Blacksburg, Virginia 24061,

United States

* Email: yaodongy@xjtu.edu.cn 


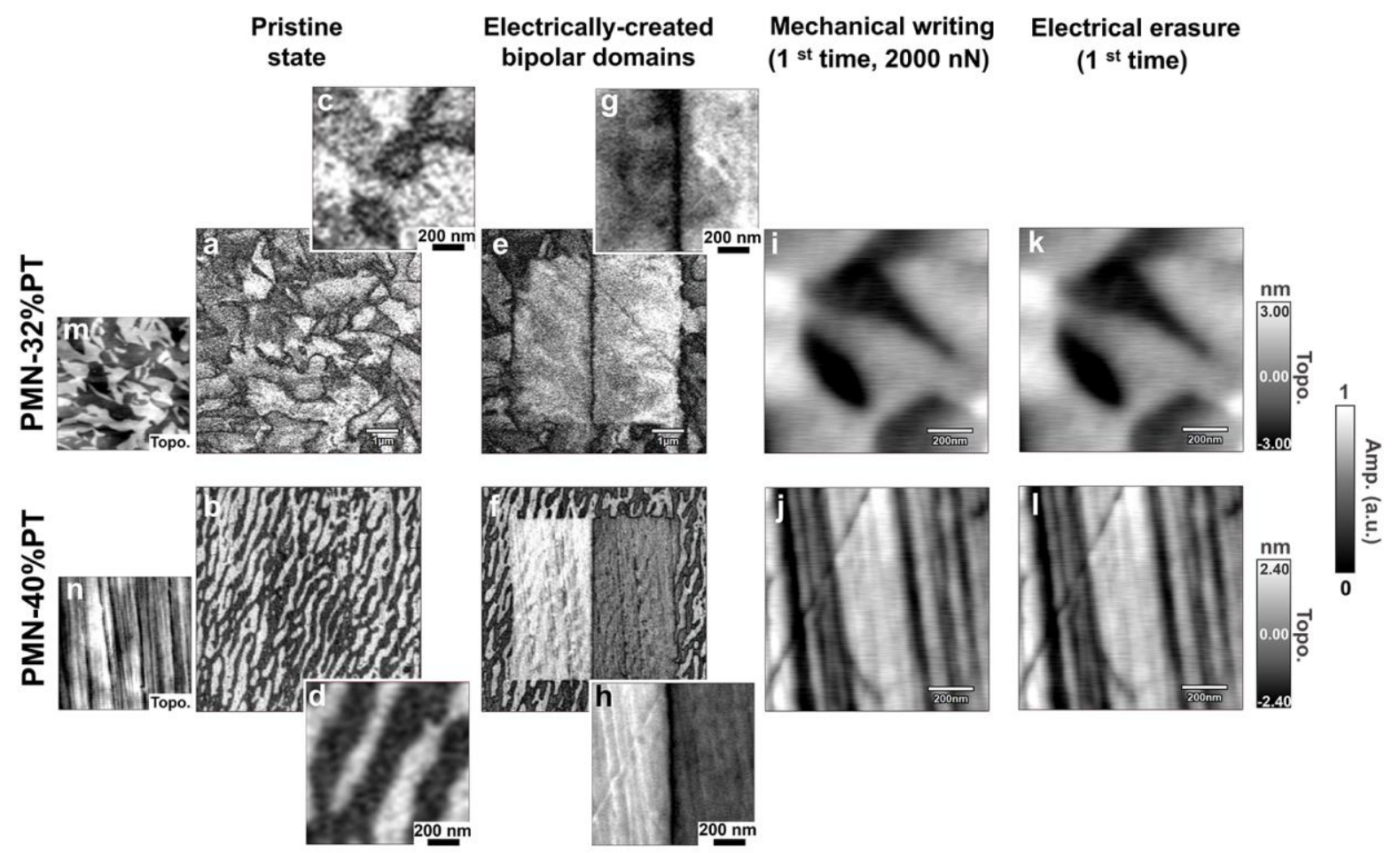

Figure S1. PFM amplitude images of the pristine domains over (a,b) $7 \mu \mathrm{m} \times 7 \mu \mathrm{m}$ and (c,d) the central $1 \mu \mathrm{m} \times 1 \mu \mathrm{m}$ in (100)-oriented PMN-32\%PT and PMN-40\%PT. (m,n) Corresponding surface topographies of the two specimens on $7 \mu \mathrm{m} \times 7 \mu \mathrm{m}$, labeled with "Topo.". PFM amplitude images of electrical-created bipolar domains on (e,f) $7 \mu \mathrm{m} \times 7 \mu \mathrm{m}$ and (g,h) $1 \mu \mathrm{m} \times$ $1 \mu \mathrm{m}$. (i-l) Surface topographies on the central $1 \mu \mathrm{m} \times 1 \mu \mathrm{m}$ after mechanical switching and electrical erasure. 

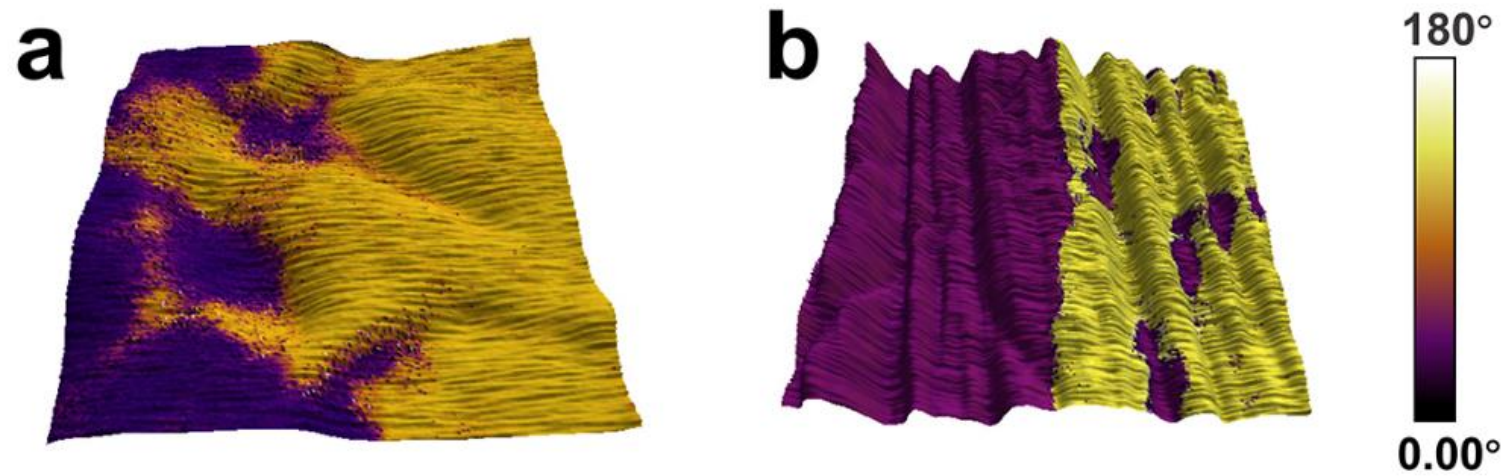

Figure S2. PFM phase response overlaid on topography images on $1 \mu \mathrm{m} \times 1 \mu \mathrm{m}$ of mechanicalinduced polarization switching by $2000 \mathrm{nN}$ in (a) PMN-32\%PT and (b) PMN-40\%PT. 


\section{PMN-32\%PT \\ State 1}

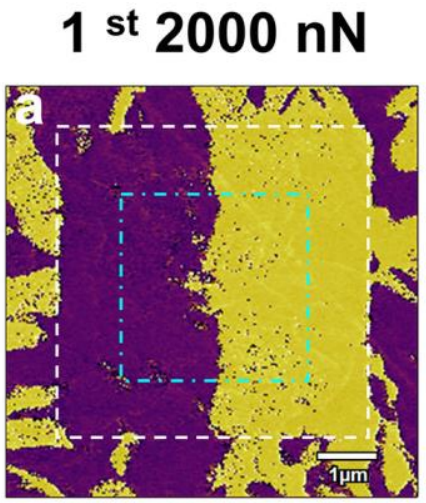

\section{2 nd $2000 \mathrm{nN}$}

\section{3 rd $2000 \mathrm{nN}$}
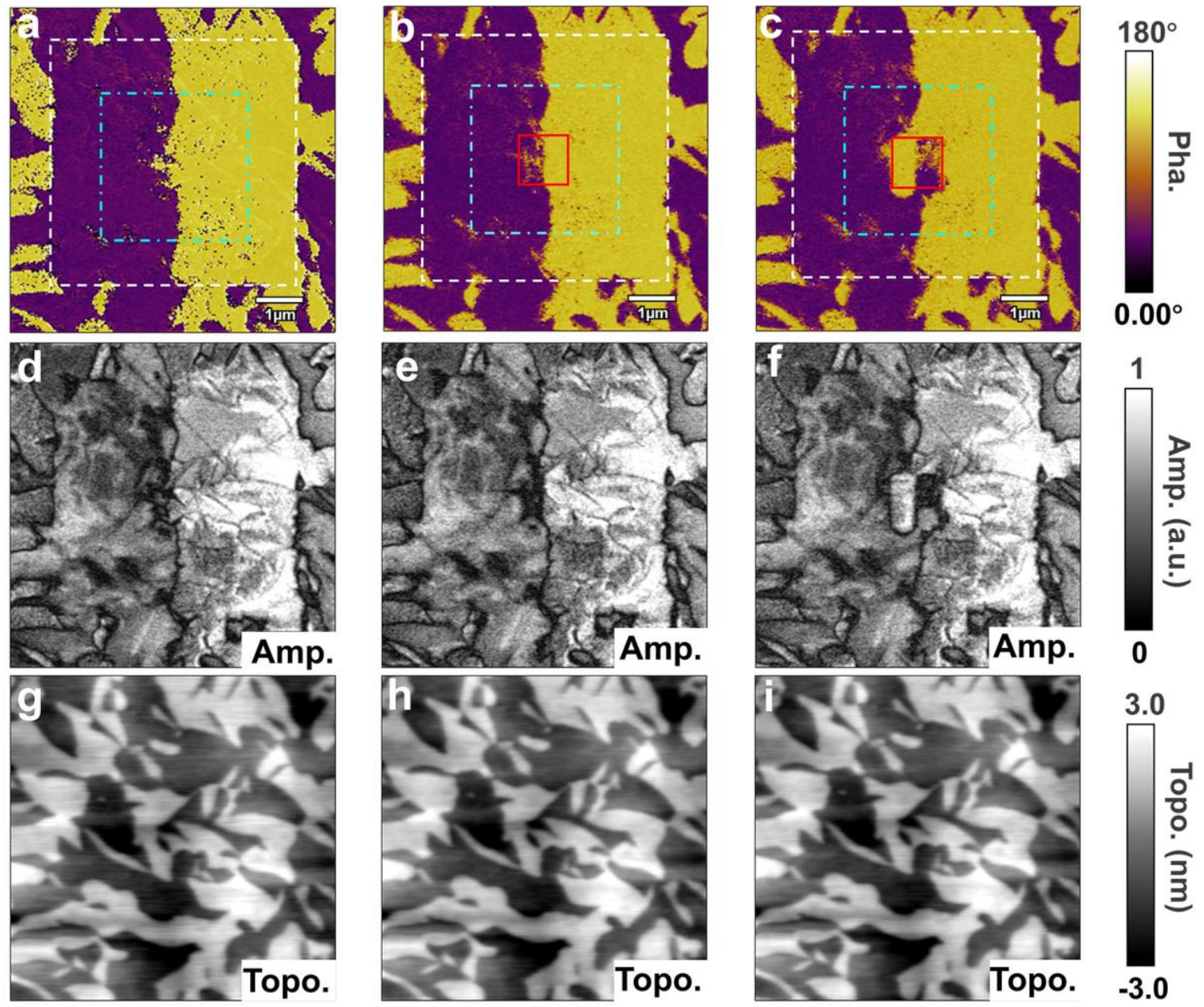

Figure S3. Overall view of in situ $7 \mu \mathrm{m} \times 7 \mu \mathrm{m}$ PFM domain pattern changes after the following mechanical switching and electrical erasure steps. The central $5 \mu \mathrm{m} \times 5 \mu \mathrm{m}$ are the electricalcreated bipolar domains (depicted in the white dashed squares). (a,d,g) Domain patterns after Step 1: the first time application of $2000 \mathrm{nN}$ on the central $3 \mu \mathrm{m} \times 3 \mu \mathrm{m}$, as shown in the cyan dot-dashed square. (b,e,h) Domain patterns after Step 2 following Step 1: the first time electrical erasure on the central $1 \mu \mathrm{m} \times 1 \mu \mathrm{m}$ (the red square), and then application of $2000 \mathrm{nN}$ for the second time on the central $1 \mu \mathrm{m} \times 1 \mu \mathrm{m}$ (the red square). (c,f,i) Domain patterns after Step 3 following Step 2: the second time electrical erasure on the central $1 \mu \mathrm{m} \times 1 \mu \mathrm{m}$ (the red 
square) and then the third time mechanical switching by $2000 \mathrm{nN}$ on the central $1 \mu \mathrm{m} \times 1 \mu \mathrm{m}$ (the red square) in PMN-32\%PT. (a-c) PFM phase images. (d-f) Corresponding PFM amplitude responses labeled with "Amp." and (g-i) corresponding AFM topographies labeled with “Topo.”. The white dashed squares depict the electrical-created bipolar regions. The cyan dotdashed squares plot the first time mechanical application regions. The red squares illustrate the first and second time electrical erasure areas and the second and third time mechanical forces loading regions. 


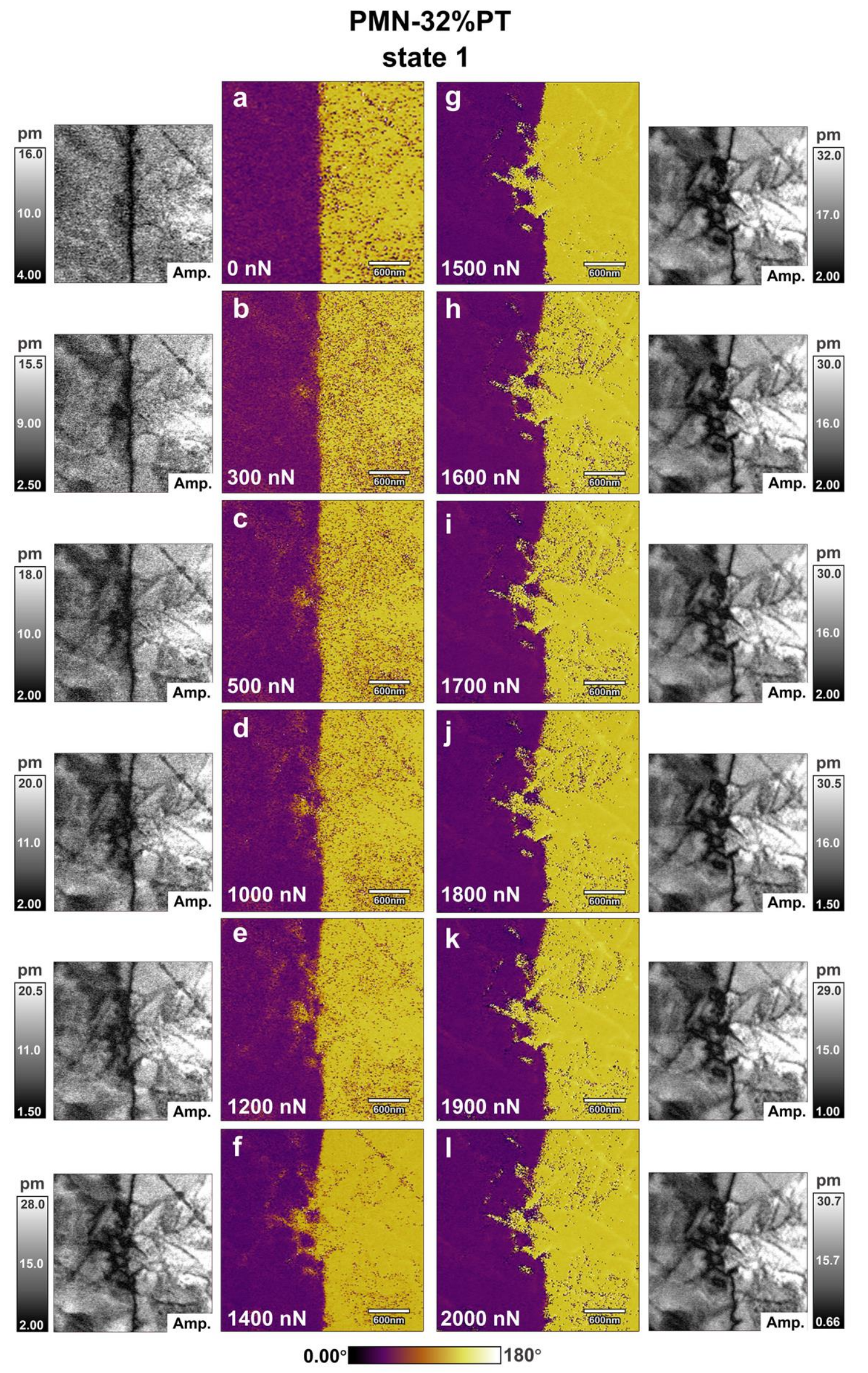


Figure S4. Evolution of mechanically switched domains by PFM under various mechanical loads (a-1) from 0 to $2000 \mathrm{nN}$ in PMN-32\%PT on the $3 \mu \mathrm{m} \times 3 \mu \mathrm{m}$ scanning region. Phase and amplitude (labeled with “Amp.") images are in color and in black and white, respectively.

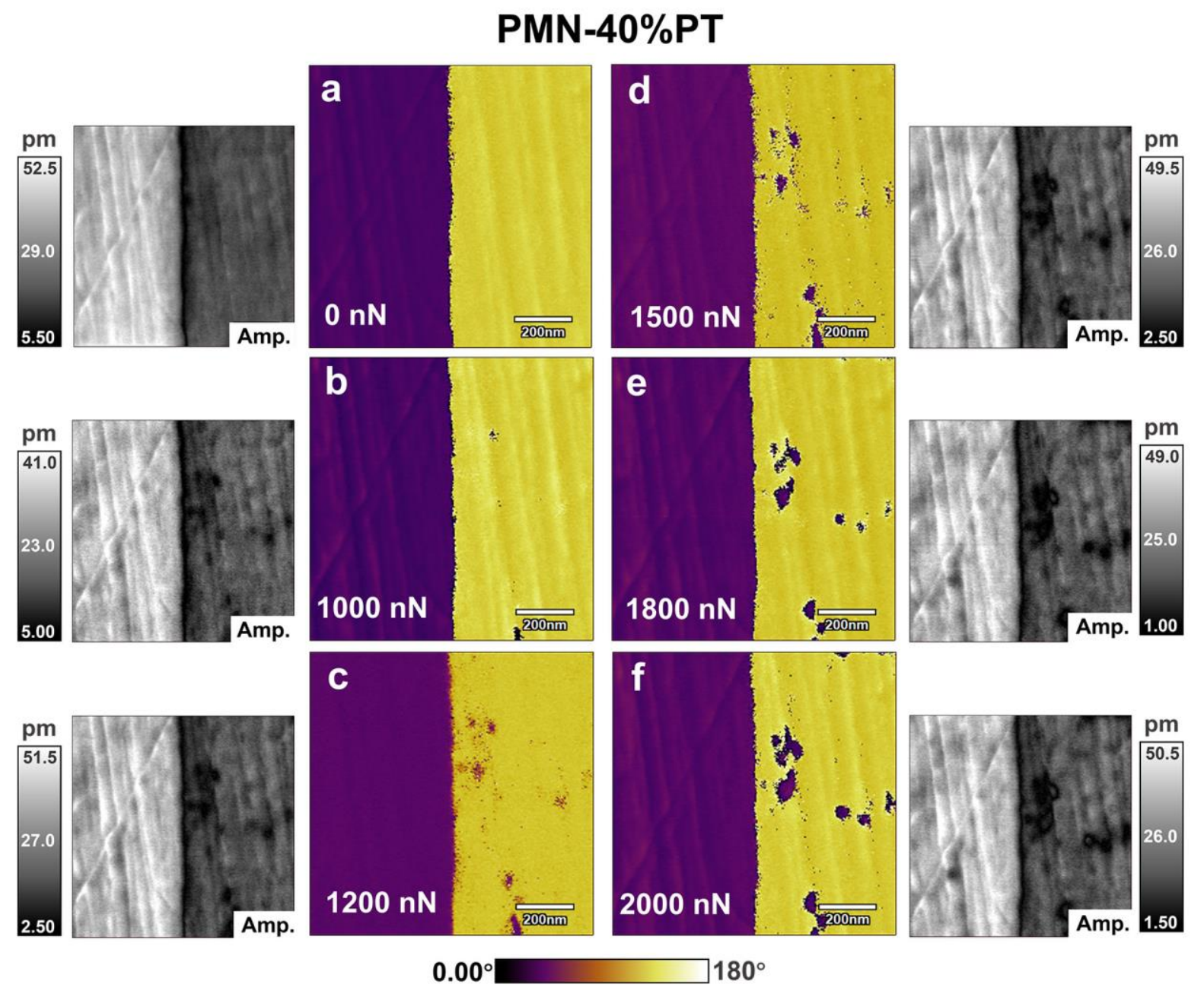

Figure S5. Evolution of mechanically switched domains by PFM under various mechanical loads (a-f) from 0 to $2000 \mathrm{nN}$ in PMN-40\%PT on the $1 \mu \mathrm{m} \times 1 \mu \mathrm{m}$ scanning region. Phase and amplitude (labeled with "Amp.") images are in color and in black and white, respectively. The PFM results under $300 \mathrm{nN}$ and $500 \mathrm{nN}$ show no difference with that under $0 \mathrm{nN}$, thus these two are not presented. 


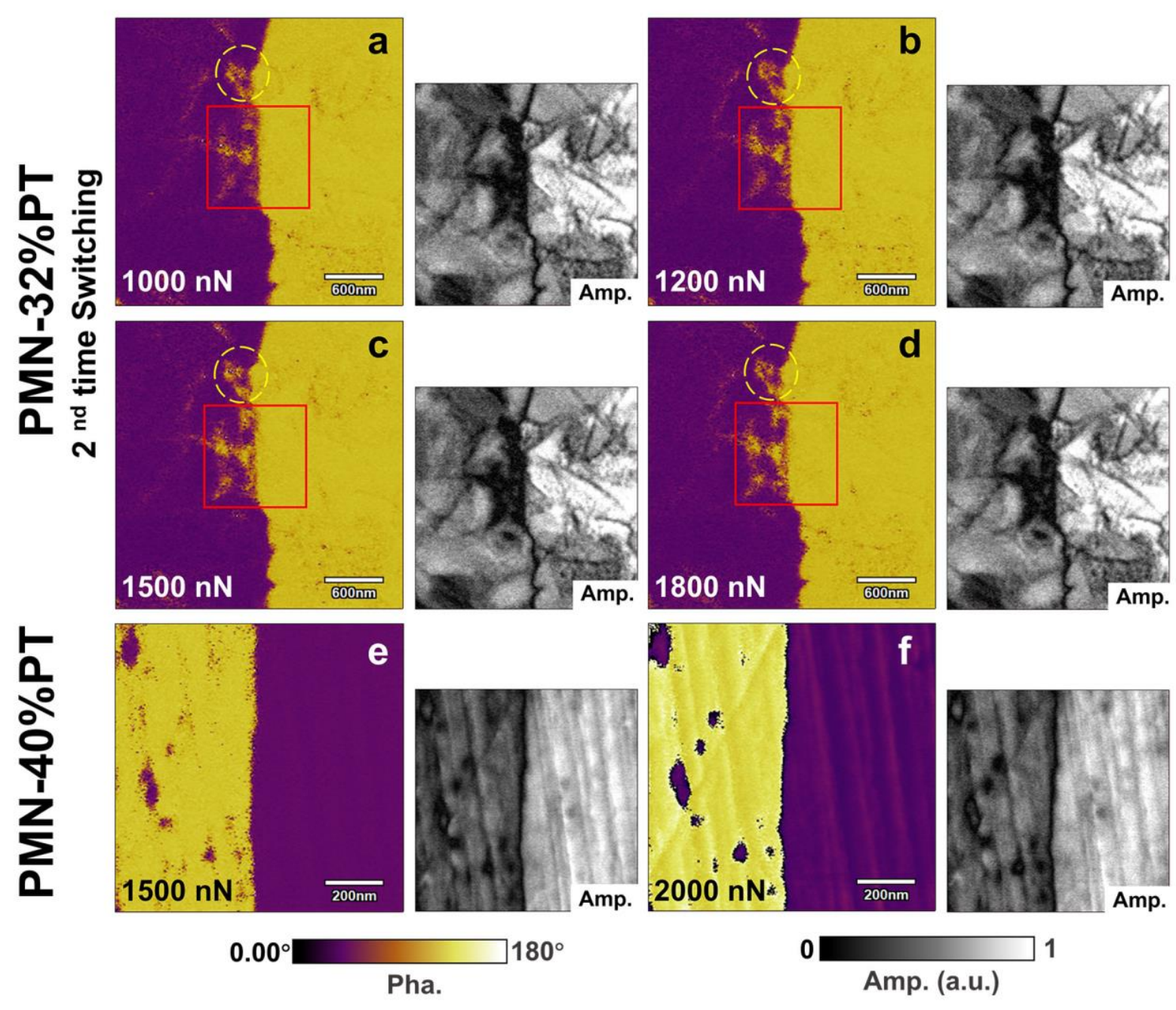

Figure S6. Evolution of mechanically switched domains by PFM in PMN-32\%PT under various mechanical loads of (a) 1000, (b) 1200, (c) 1500 and (d) $1800 \mathrm{nN}$ applied for the second time. Evolution of the third time mechanical-switched domains by PFM in PMN-40\%PT under (e) 1500 and (f) 2000 nN. PFM phase and amplitude (labeled with "Amp.”) images are in color and in black and white, respectively. Red squares in (a-d) illustrate the second time force application region. Yellow dashed circles in (a-d) show that the first time mechanical-switched domains remain the same during the second time switching process. 


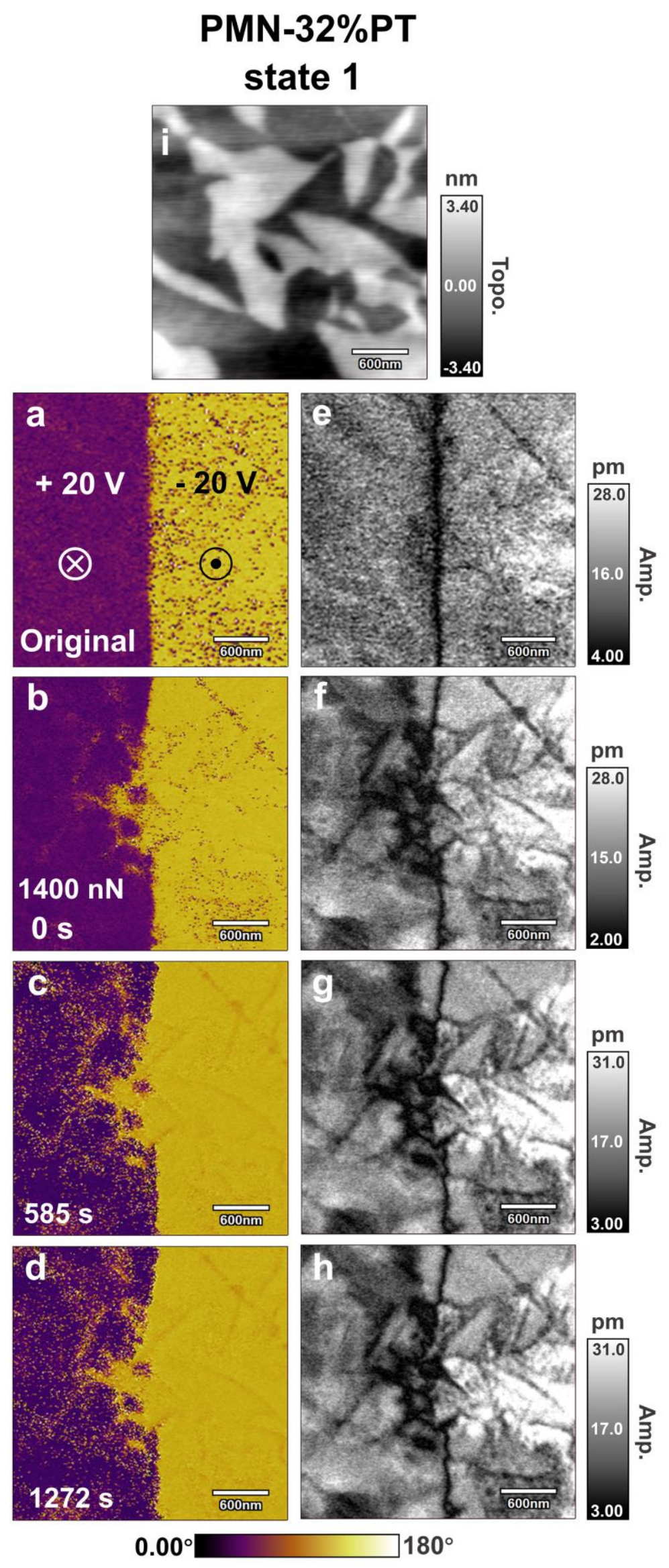


Figure S7. Retention results of the mechanical-induced switched domains by $1400 \mathrm{nN}$ in PMN$32 \% \mathrm{PT}$ state 1 on $3 \mu \mathrm{m} \times 3 \mu \mathrm{m}$ taking the first PFM image after withdrawal of the mechanical force as "0 s". (a,e) The original electrical-created bipolar domain pattern. Domain patterns after mechanical writing taken from (b,f) 0 to $(\mathrm{c}, \mathrm{g}) 585 \mathrm{~s}$, then to $(\mathrm{d}, \mathrm{h}) 1272 \mathrm{~s}$. (a-d) PFM phase images. (e-h) Their corresponding amplitude responses. (i) Surface topography of these domains which remains the same during the process.

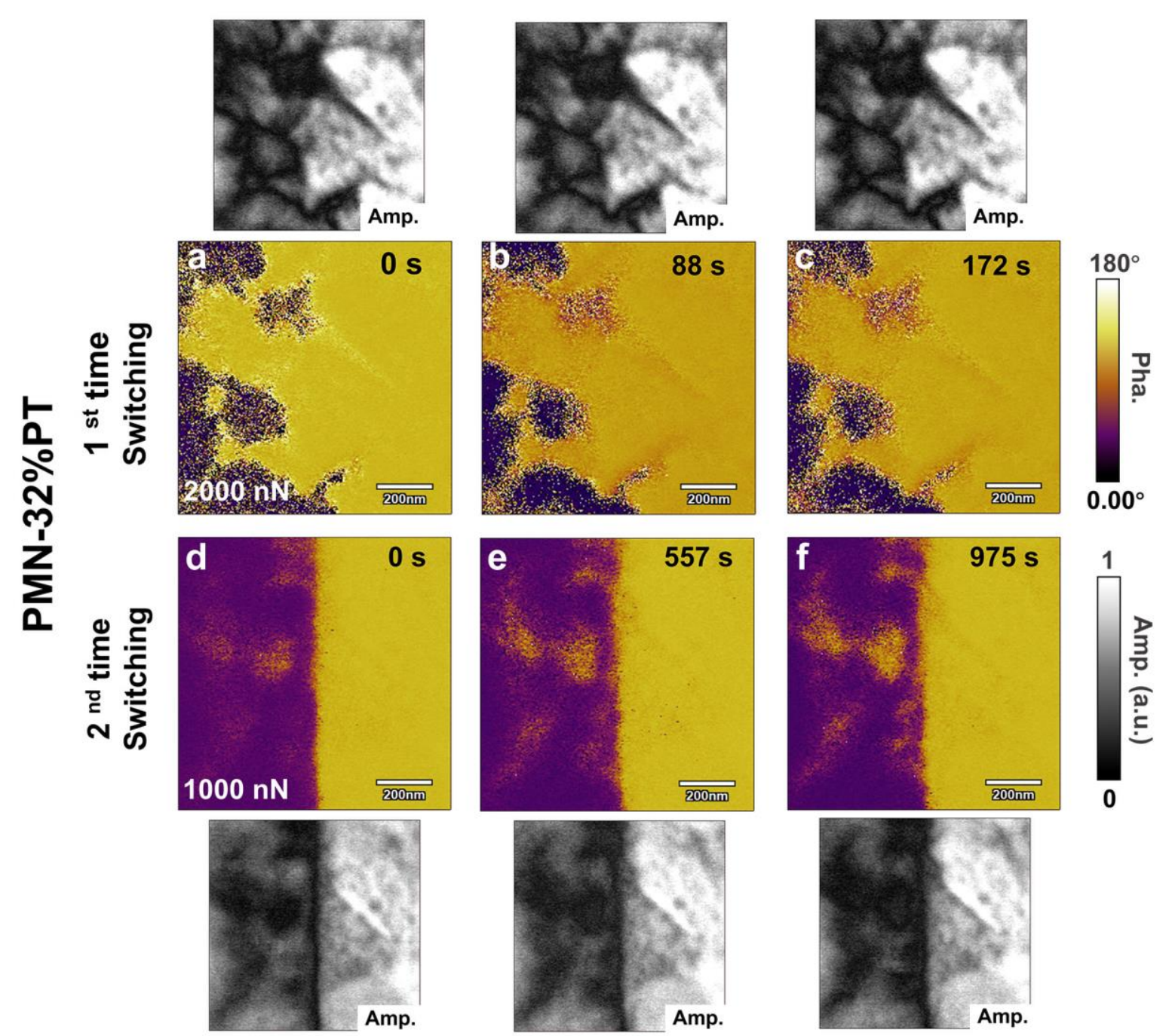

Figure S8. In situ retention results of (a-c) the first time by $2000 \mathrm{nN}$ and (d-f) the second time by $1000 \mathrm{nN}$ mechanical-induced switched domains in PMN-32\%PT (state 1 ) on $1 \mu \mathrm{m} \times 1 \mu \mathrm{m}$, 
taking the first PFM image after withdrawal of the mechanical force as "0 s". PFM phase images are shown in color, and amplitude images are in black and white labeled with "Amp.". 


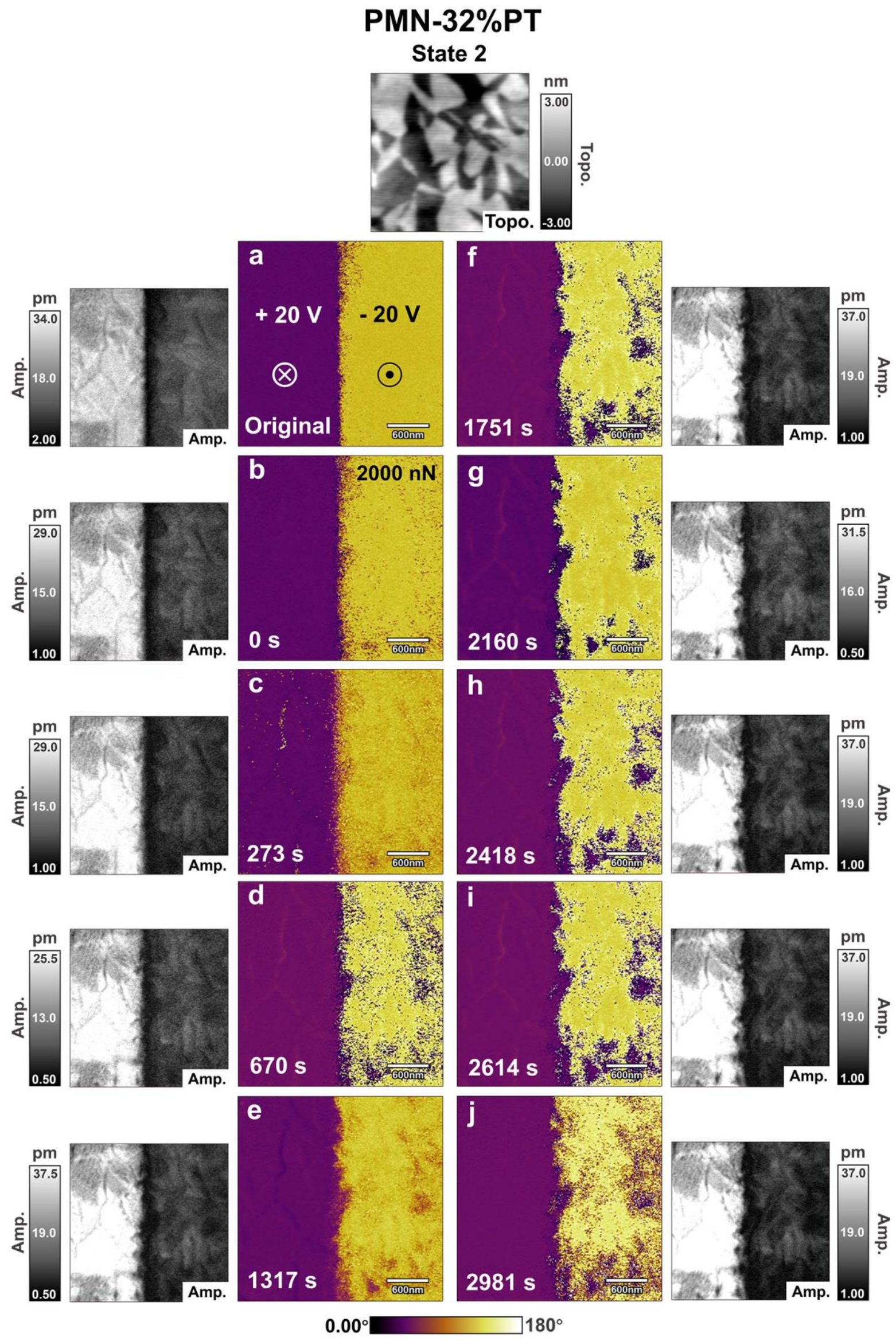


Figure S9. Time-dependent evolution of mechanically switched domains in PMN-32\%PT (state 2) on $3 \mu \mathrm{m} \times 3 \mu \mathrm{m}$, taking the first PFM image after withdrawal of the mechanical force as "0 s". (a) The original electrical-created bipolar domain pattern. (b-j) In situ domain evolution after mechanical writing by $2000 \mathrm{nN}$ from the SPM tip taken at various time from 0 to $2981 \mathrm{~s}$. PFM phase images are shown in color, and amplitude and topography images are in black and white and labeled with "Amp." and "Topo.", respectively. 


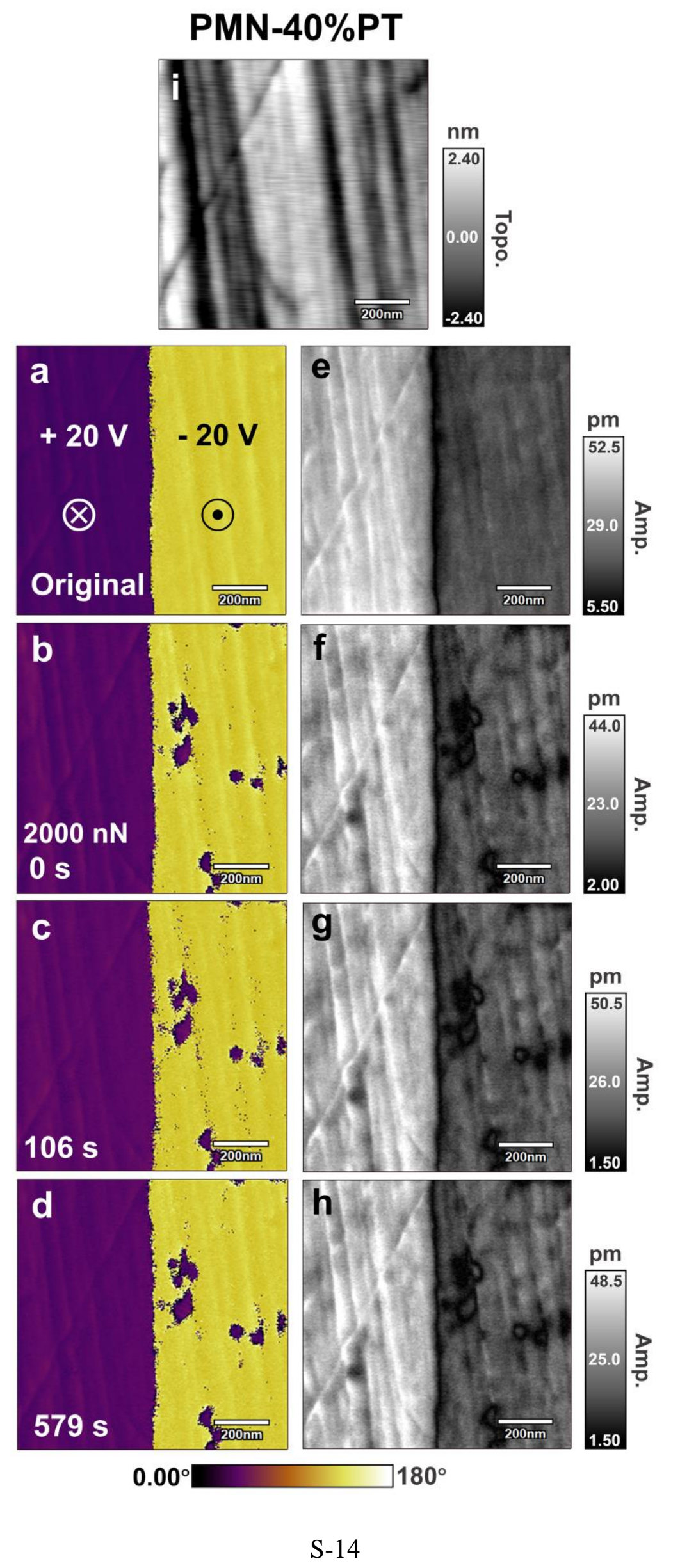


Figure S10. Time-dependent evolution of the mechanical-induced switched domains in PMN$40 \% \mathrm{PT}$ on $1 \mu \mathrm{m} \times 1 \mu \mathrm{m}$, taking the first PFM image after withdrawal of the mechanical force as " $0 \mathrm{~s}$ ". (a,e) The original electrical-created bipolar domain pattern. In situ domain evolution after mechanical writing by $2000 \mathrm{nN}$ from the SPM tip taken at various time from $(\mathrm{b}, \mathrm{f}) 0$ to $(\mathrm{c}, \mathrm{g}) 106 \mathrm{~s}$, and then to (d,h) 579 s. (a-d) PFM phase images are shown in color, and (e-h) amplitude and (i) topography images are in black and white and labeled with "Amp." and “Topo.”, respectively. 